\title{
Peripartum cardiomyopathy with recovery in a patient with coincidental Eisenmenger ventricular septal defect
}

\author{
Celia M Oakley, Petros Nihoyannopoulos
}

\begin{abstract}
Severe pulmonary vascular disease was found during pregnancy in a 31 year old woman with a large ventricular septal defect. Three months after delivery peripartum cardiomyopathy with severe biventricular failure developed. This was a chance association. Recovery of ventricular function was slow but was complete two years later. There was no advance in the pulmonary vascular disease.
\end{abstract}

\section{Case report}

A 31 year old Indian woman was found to have a systolic murmur in the antenatal clinic during the tenth week of her first pregnancy. She gave no cardiac history and did not report any limitation of exercise capacity. She was referred to the cardiology clinic where she was found to be acyanotic with normal venous pressure, right ventricular heave, pulmonary ejection click, and loud "single" second sound-signs of pulmonary hypertension associated with a large ventricular septal defect. The absence of cyanosis implied that the shunt was dominantly left to right. Chest $x$ ray showed that her heart was only slightly increased by enlargement of the right atrium and of the proximal pulmonary arteries (fig 1A). The electrocardiogram showed sinus rhythm with right axis deviation and right ventricular hypertrophy. Cross sectional and Doppler echocardiography showed a large subaortic ventricular septal defect with obligatory pulmonary hypertension at systemic level. Both the left and right ventricles showed normal function; neither was enlarged (fig 2). There was little evidence of flow and no turbulence across the ventricular septal defect. Despite some concern about the risk to the mother of accelerating pulmonary vascular disease, her pregnancy proceeded uneventfully except for the development of pregnancy diabetes needing insulin. She had a haemoptysis at 35 weeks attributed to the pulmonary hypertension ("pulmonary apoplexy"). At that time oximetry showed $95 \%$ saturation, her arterial $\mathrm{PO}_{2}$ was $11.0 \mathrm{kPa}$ and the chest $x$ ray was clear. A healthy female infant weighing $2 \cdot 8 \mathrm{~kg}$ was delivered by elective lower segment caesarean section at 37 weeks. There were no complications. She was well on discharge and the physical signs were unchanged.
She remained well for three months after delivery but then became increasingly short of breath with cyanosis and swelling of her legs and hands. Physical examination showed new intense central and peripheral cyanosis. The blood pressure was $80 / 60 \mathrm{mmHg}$, her jugular venous pressure was raised at $8 \mathrm{~cm}$ and she had developed an apical third heart sound with a loud pansystolic murmur at the left sternal edge and apex attributed to atrioventricular valve regurgitation. Her chest $x$ ray showed that her heart had become enlarged (fig 1B). There was now a right ventricular "strain" pattern on the electrocardiogram with $T$ inversion. Her echocardiogram showed new global impairment of right and left ventricular function (fig 2). At cardiac catheterisation (table) there was bidirectional but predominantly right to left shunting at ventricular level $(Q p)$ Qs $=0.76: 1$ ) with equal left and right ventricular pressures and pulmonary hypertension at the systemic level. After inhalation of a high inspired oxygen content for ten minutes there was a shift to predominantly left to right shunting with a rise in aortic saturation from 75 to $89 \%(\hat{\mathrm{p}} / \mathrm{Q} \mathrm{s}=1 \cdot 17: 1)$, implying a degree of reactivity of the pulmonary vascular bed. Angiography confirmed the large ventricular septal defect. There was severe global impairment of right and left ventricular function. Her coronary arteries were normal. Three biopsy specimens were taken of the left ventricle. Histological examination (including electron microscopy) showed no recognisable abnormality. Viral studies and tests for autoantibodies were negative and thyroid function and vanillyl mandelic acids were normal.

Peripartum cardiomyopathy was diagnosed. Despite treatment with digoxin, diuretics, and captopril her condition remained unchanged and she was referred and accepted for heartlung transplantation. Immunosuppressive treatment was not used. ${ }^{1}$ In the months that followed a retinal vein thrombosis with secondary glaucoma developed and warfarin treatment was started. She continued to be hypotensive with low output failure, raised venous pressure, and tricuspid regurgitation and was unable to do any housework or look after her child. She was seen frequently in the clinic and it was not until a year after the birth that she began to improve. This improvement was confirmed by serial chest $x$ ray (fig 1 ) and echocardiography (fig 2). Over the course of 
Figure 1 Chest she was first seen during of heart failure $(B)$, and after recovery (C). $A$ and $C$ show an only slightly enlarged heart with evidence of severe

pulmonary hypertension right shunt. $B$ shows gross cardiomegaly. radiographs taken when pregnancy $(A)$, after onset but little evidence of left to
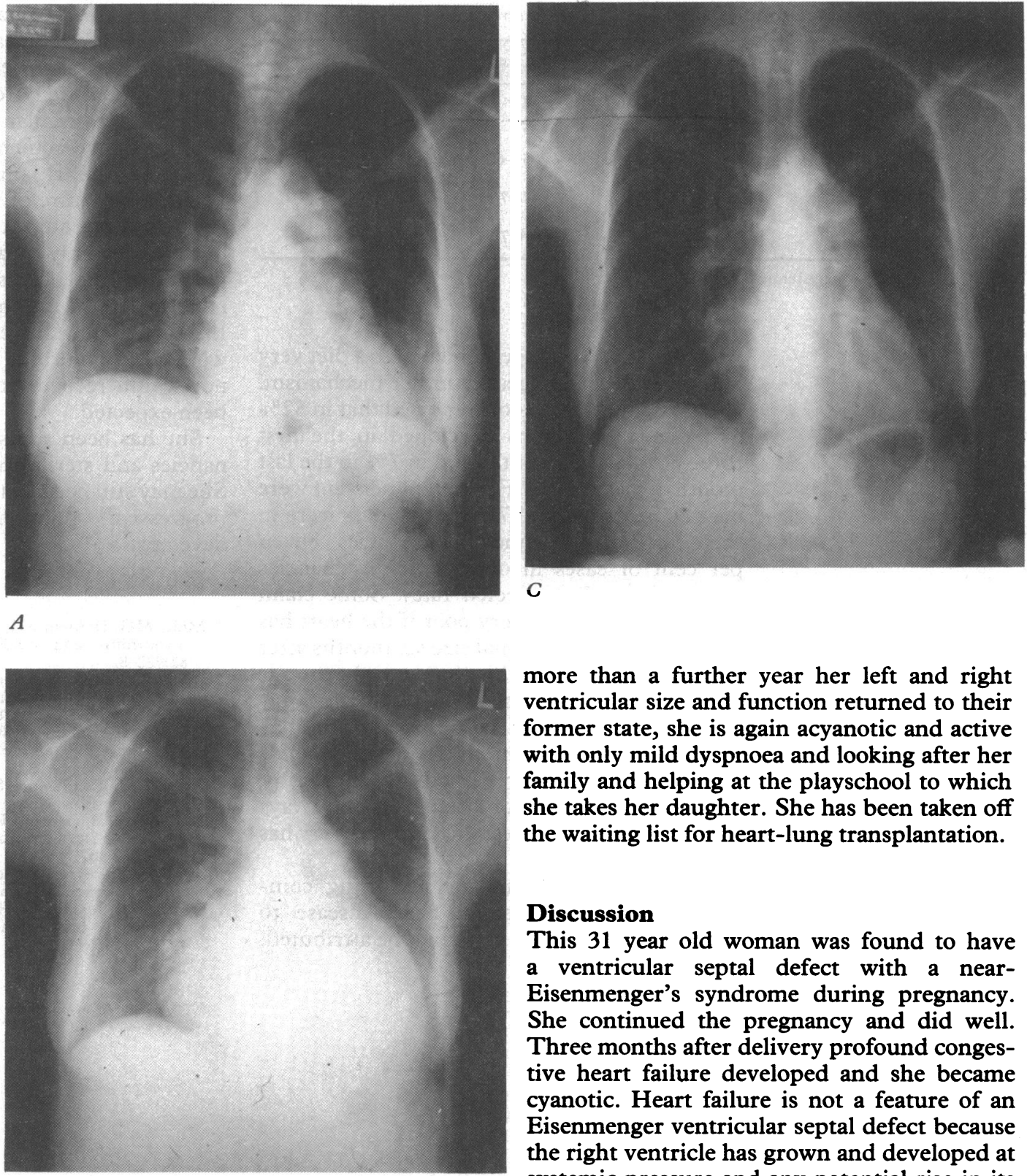

14.11.86

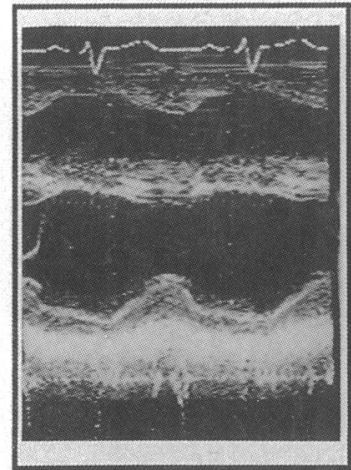

LVd/s (mm) 45/23

FS (\%)
23.7.87

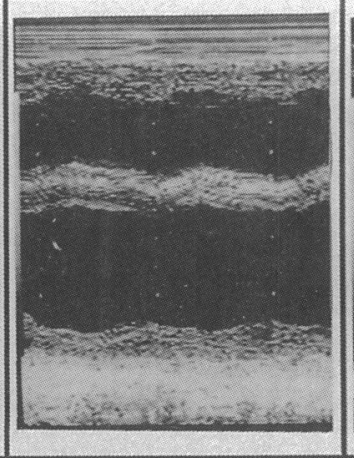

$48 / 42$

13
18.5.90

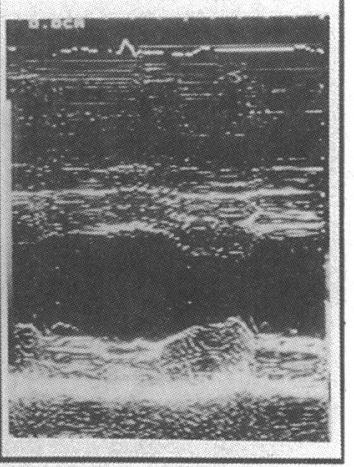

$41 / 25$

39
Figure 2 Serial $M$ mode echocardiographic recordings showing good left and right ventricular function on 14 November 1986 but greatly reduced biventricular function on 23 July 1987 with some dilatation. The record of 18 May 1990 showed that ventricular function had recovered. The hypertrophied right ventricle is best seen in the first two records. LV, left ventricular end diastolic (d) and end systolic(s) dimensions; $F S$, fractional shortening.
$C$

more than a further year her left and right ventricular size and function returned to their former state, she is again acyanotic and active with only mild dyspnoea and looking after her family and helping at the playschool to which she takes her daughter. She has been taken off the waiting list for heart-lung transplantation.

\section{Discussion}

This 31 year old woman was found to have a ventricular septal defect with a nearEisenmenger's syndrome during pregnancy. She continued the pregnancy and did well. Three months after delivery profound congestive heart failure developed and she became cyanotic. Heart failure is not a feature of an Eisenmenger ventricular septal defect because the right ventricle has grown and developed at systemic pressure and any potential rise in its systolic or diastolic pressure is immediately dissipated by shunting across the large ventricular septal defect. Myocardial failure is therefore not part of the course. Consequently, we believe that in our patient deterioration in biventricular function was caused by the development of a peripartum cardiomyopathy and was not associated with the ventricular septal defect and pulmonary hypertension.

Peripartum cardiomyopathy was first described by Ritchie in $1849 .^{2}$ The diagnosis is made when heart failure develops in the last month of pregnancy or in the first five postnatal months in the absence of a confirmed aetiology or of demonstrable heart disease before the last month of pregnancy. ${ }^{34}$ The aetiology is unclear but it is probably immunological. Biopsy specimens taken early in the course of the disease have sometimes shown inflammatory changes suggesting a viral or autoimmune mechanism. ${ }^{135}$ Poor nutrition has been suggested $^{3}$ though most women affected are well nourished. Pre-eclampsia was seen in a disproportionate number of pregnancies, ${ }^{3}$ and the very high incidence of heart failure in pregnant 
Data from cardiac catheterisation after onset of heart failure

\begin{tabular}{|c|c|c|c|}
\hline Data & Pressures $(\mathrm{mm} \mathrm{Hg}$ ) & $\mathrm{O}_{2}$ sat $(\%)$ & After $\mathrm{O}_{2}$ \\
\hline Right atrium & 20 & 41 & 55 \\
\hline Right ventricle & $\frac{120}{24}$ & 57 & 67 \\
\hline Pulmonary artery & $\frac{110}{55} \quad-86$ & $53-62$ & 64 \\
\hline Aorta & $\frac{110}{70}$ & $76 \cdot 75$ & 89 \\
\hline QP:QS & & $0 \cdot 76: 1$ & $1 \cdot 17: 1$ \\
\hline
\end{tabular}

QP:QS = pulmonary:stystemic flow ratio.

women in parts of Nigeria related to a diet very high in sodium ${ }^{6}$ suggests another mechanism. Demakis and Rahimtoola reported that in $82 \%$ of patients symptoms developed in the first three postpartum months and in $7 \%$ in the last month of pregnancy. ${ }^{4}$ Forty eight per cent were over 30 years old and $71 \%$ of patients were in their third or subsequent pregnancies. Seven per cent of cases involved twin pregnancies (seven times the expected rate). Some claim that the prognosis is very poor if the heart has not returned to its normal size six months after the diagnosis; ${ }^{7}$ but this has not been our experience. As our case shows, recovery may start later and take as long as two years. The outlook for subsequent pregnancies depends on whether the heart returned to normal after the first episode ${ }^{7}$ the condition does not always recur in subsequent pregnancies if recovery has been complete.

Our patient was unusual in having coincidental severe congenital heart disease to which the heart failure could not be attributed.
She was also unusual in remaining in heart failure (New York Heart Association Class IIIIV) for a year postpartum before she started to improve and then recovering her pre-pregnancy cardiovascular status in the subsequent year. The development of central cyanosis after the onset of heart failure can be explained by a greater admixture of venous blood during the period of low output failure. If cyanosis had been caused only by an increase in pulmonary vascular resistance associated with the pregnancy then, as in the natural history of an Eisenmenger ventricular septal defect, she would have gone blue but not into heart failure, nor would recovery of her former state have been expected.

She has been advised against further pregnancies and sterilisation has been performed. She may still need heart/lung transplantation if progressive pulmonary vascular disease develops.

1 Midei MG, DeMent SH, Feldman AM, et al. Peripartum myocarditis and cardiomyopathy. Circulation 1990; 81:922-8.

2 Ritchie C. Clinical contributions to the pathology, diagnosis and treatment of certain chronic disease of the heart. and treatment of certain chronic

3 Walsh J, Burch G, Black W, Ferrons V, Hibbs R. Idiopathic myocardiopathy of the puerperium. Circulation 1965 32:19-31.

4 Demakis J, Rahimtoola S. Peripartum cardiomyopathy. Circulation 1971;44:964-8.

5 Melvin KR, Richardson P, Olsen E, Daly K, Jackson G Peripartum cardiomyopathy due to myocarditis. $N$ Engl Med 1982;307:731-4.

6 Seftel H, Susser M. Maternity and myocardial failure in African women. Br Heart J 1961;23:43-52.

7 Demakis J, Shahbuden H, Rahimtoola S, et al. Natural course of peripartum cardiomyopathy. Circulation 1971;44:1053-61. 